\title{
MARVEL Analysis of the Measured High-resolution Rovibronic Spectra of the Calcium Monohydroxide Radical $(\mathrm{CaOH})$
}

\author{
Yixin Wang $^{1,2}$ (iD, Alec Owens ${ }^{1}$ (10), Jonathan Tennyson ${ }^{1}$ (D), and Sergei N. Yurchenko ${ }^{1}$ (1) \\ ${ }^{1}$ Department of Physics and Astronomy, University College London, Gower Street, WC1E 6BT London, UK; s.yurchenko@ucl.ac.uk \\ ${ }^{2}$ Nankai University, 94 Weijin Road, Tianjin, People's Republic of China \\ Received 2019 December 22; revised 2020 February 26; accepted 2020 March 25; published 2020 April 30
}

\begin{abstract}
The calcium monohydroxide radical $(\mathrm{CaOH})$ is an important astrophysical molecule relevant to cool stars and rocky exoplanets, among other astronomical environments. Here, we present a consistent set of highly accurate rovibronic (rotation-vibration-electronic) energy levels for the five lowest electronic states $\left(\tilde{X}^{2} \Sigma^{+}, \tilde{A}^{2} \Pi, \tilde{B}^{2} \Sigma^{+}\right.$, $\tilde{C}^{2} \Delta$, and $\tilde{D}^{2} \Sigma^{+}$) of $\mathrm{CaOH}$. A comprehensive analysis of the published spectroscopic literature on this system has allowed 1955 energy levels to be determined from 3204 rovibronic experimental transitions, all with unique quantum number labeling and measurement uncertainties. The data set covers rotational excitation up to $J=62.5$ for molecular states below $29,000 \mathrm{~cm}^{-1}$. The analysis was performed using the Measured Active RotationalVibrational Energy Levels algorithm, which is a robust procedure based on the theory of spectroscopic networks. The data set provided will significantly aid future interstellar, circumstellar, and atmospheric detections of $\mathrm{CaOH}$, as well as assist in the design of efficient laser cooling schemes in ultracold molecule research and precision tests of fundamental physics.
\end{abstract}

Unified Astronomy Thesaurus concepts: Molecular spectroscopy (2095); Exoplanet atmospheres (487); Astronomy databases (83)

Supporting material: FITS files

\section{Introduction}

The calcium monohydroxide radical $\left({ }^{40} \mathrm{Ca}^{16} \mathrm{O}^{1} \mathrm{H}\right)$ is a linear triatomic molecule of increasing astronomical interest due to its expected presence in the atmospheres of hot rocky super-Earth exoplanets (Bernath 2009; Tennyson \& Yurchenko 2017). This class of exoplanets is very close to their host star and tidally locked, with their dayside exposed to extremely high temperatures, e.g., $2000-4000 \mathrm{~K}$. The material present on the surface of the planet, including rock-forming elements such as silicon, magnesium, iron, calcium, and so on, will vaporize to some extent and produce an atmosphere strongly dependant on planetary composition (Schaefer et al. 2012; Fegley et al. 2016). Investigating the spectroscopy of hot rocky super-Earths requires accurate spectroscopic data on simple molecules composed of rock-forming elements, like $\mathrm{CaOH}$. However, data for $\mathrm{CaOH}$ is not necessarily available or easily accessible. For example, a recent systematic study modeling M-dwarf photospheres by Rajpurohit et al. (2013) noted missing opacity from the benchmark BT-Settl model due to three molecules: $\mathrm{NaH}, \mathrm{AlH}$, and $\mathrm{CaOH}$. The ExoMol project has since computed line lists for $\mathrm{NaH}$ (Rivlin et al. 2015) and $\mathrm{AlH}$ (Yurchenko et al. 2018), meaning that $\mathrm{CaOH}$, notably its band around $18,000 \mathrm{~cm}^{-1}$, remains as the only identified missing source of opacity in these objects.

Given the high cosmic abundance of calcium with respect to molecular hydrogen, it is reasonable to expect calcium-bearing molecules such as $\mathrm{CaOH}$ in other interstellar and circumstellar environments. For example, a possible formation mechanism in the interstellar medium is through the reaction of $\mathrm{Ca}^{+}$ions with small oxide interstellar grains to release gas-phase $\mathrm{CaOH}$ (Duley \& Millar 1978). Tsuji (1973) predicted that $\mathrm{CaOH}$ would be the most abundant calcium-bearing molecule in oxygen-rich late-type stars at temperatures of $T=1000-2000 \mathrm{~K}$, while the
$\tilde{B}^{2} \Sigma^{+}-\tilde{X}^{2} \Sigma^{+}$electronic band of $\mathrm{CaOH}$ was tentatively assigned in the spectra of late-type M-dwarf stars (Pesch 1972).

A large number of experimental studies have measured the rovibronic (rotation-vibration-electronic) spectrum of $\mathrm{CaOH}$; however, there is no centralized source containing this information, aside from the Cologne Database for Molecular Spectroscopy (CDMS) database (Müller et al. 2001, 2005; Endres et al. 2016) but this only covers the microwave region $\left(0-34 \mathrm{~cm}^{-1}\right)$. In this work, we present a data set of highly accurate molecular rovibronic transitions and energy levels for the $\mathrm{CaOH}$ molecule, obtained by evaluating all available spectroscopic data on $\mathrm{CaOH}$ from the published literature using the Measured Active Rotational-Vibrational Energy Levels (MARVEL) algorithm (Császár et al. 2007; Furtenbacher et al. 2007; Furtenbacher \& Császár 2012; Tóbiás et al. 2019). This procedure takes a set of assigned transition frequencies with measurement uncertainties and converts them into a consistent set of empirical energy levels, each with their own measurement uncertainty and unique quantum numbers and state labels.

A MARVEL data set for $\mathrm{CaOH}$ will considerably aid future astronomical detection of this molecule, particularly because of its large wavelength and rotational excitation coverage. Furthermore, it will benefit the calculation of a molecular line list for $\mathrm{CaOH}$, which is currently being undertaken by the ExoMol project (Tennyson \& Yurchenko 2012; Tennyson et al. 2016). MARVEL data sets of empirical energy levels can greatly improve the accuracy of computed molecular opacities, as was done for the recent MARVEL titanium oxide data set (McKemmish et al. 2017) and TiO line list (McKemmish et al. 2019), whose detection in exoplanet atmospheres had been hampered by the inaccuracy of line positions in the available line lists (Hoeijmakers et al. 2015).

We mention that the alkaline earth monohydroxide radicals, including $\mathrm{CaOH}$ (Augustovičová \& Bohn 2019; 


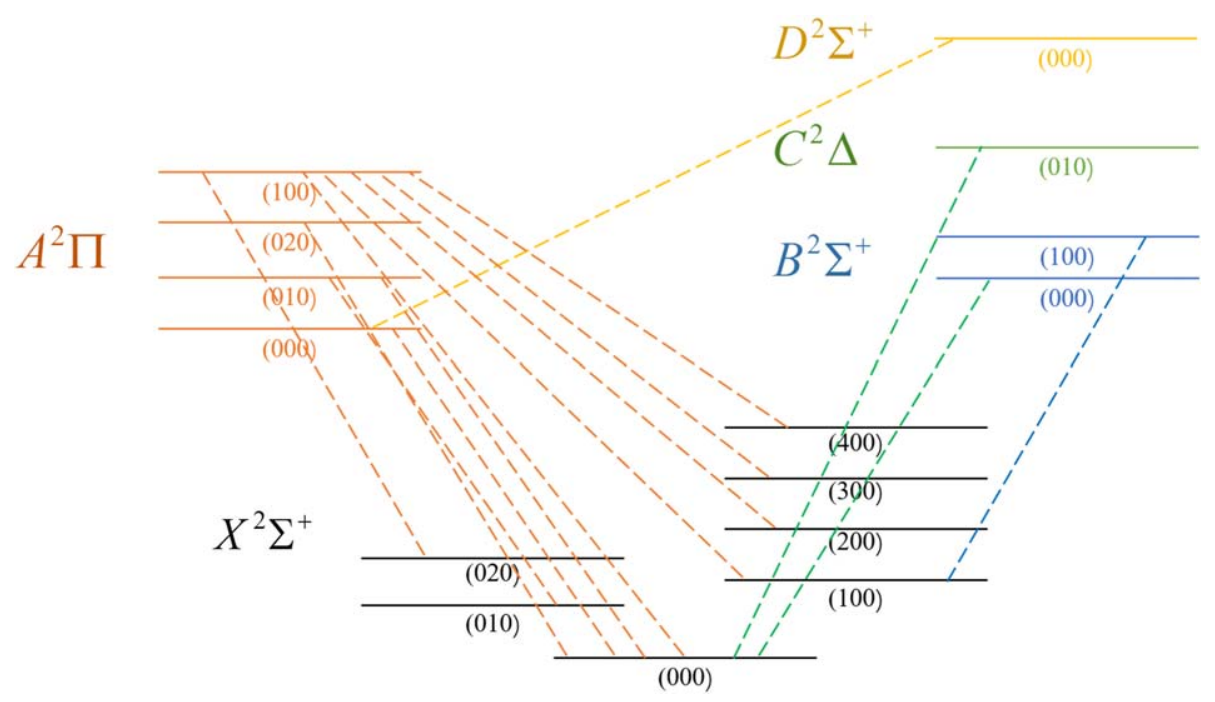

Figure 1. Rovibronic states and transitions of $\mathrm{CaOH}$ considered in this work.

Kozyryev et al. 2019), are relevant in studies of ultracold molecules and precision tests of fundamental physics due to their favorable energy level structure. A list of highly accurate energy levels across multiple electronic states can be useful in this field, especially for the design of efficient laser cooling schemes which requires knowledge of molecular rovibronic structure to a high degree of accuracy.

\section{Theoretical Background}

\subsection{The MARVEL Approach}

The MARVEL procedure (Császár et al. 2007; Furtenbacher et al. 2007; Furtenbacher \& Császár 2012; Tóbiás et al. 2019) is based on the theory of spectroscopic networks (Császár \& Furtenbacher 2011; Furtenbacher \& Császár 2012; Furtenbacher et al. 2014; Árendás et al. 2016) and offers an elegant way to construct and represent complex networks such as those contained in a molecule's spectroscopy. Energy levels are represented as nodes with the allowed transitions linking them and the corresponding transition intensities acting as weights. Provided with a data set of assigned transitions with measurement uncertainties, MARVEL will produce a consistent set of uniquely labeled empirical-quality energy levels, with the uncertainties propagated from the input transitions to the output energies.

From a user perspective, transitions included in the MARVEL data set must have a measurement uncertainty and every energy level has to be uniquely labeled, typically by a set of quantum numbers as discussed below. The chosen set of quantum numbers must be consistent across the whole data set but need not be physically meaningful. However, a sensible choice will benefit comparisons with other data and allow the final data set to be readily utilized in future studies. MARVEL is publicly available through a user-friendly web interface at http://kkrk.chem.elte.hu/marvelonline and numerous MARVEL studies have been performed on astronomically important diatomic and small polyatomic molecules: $\mathrm{NH}_{3}$ (Al Derzi et al. 2015), $\mathrm{C}_{2}$ (Furtenbacher et al. 2016), TiO (McKemmish et al. 2017), $\mathrm{C}_{2} \mathrm{H}_{2}$ (Chubb et al. 2018a), $\mathrm{H}_{2} \mathrm{~S}$ (Chubb et al. 2018b), $\mathrm{ZrO}$ (McKemmish et al. 2018), NH (Darby-Lewis et al. 2019), $\mathrm{SO}_{2}$ (Tóbiás et al. 2018), $\mathrm{H}_{3}^{+}$(Furtenbacher et al. 2013b) and its isotopologues (Furtenbacher et al. 2013a), and $\mathrm{H}_{2}{ }^{16} \mathrm{O}$ and its isotopologues (Tennyson et al. 2009, 2010, 2013, 2014a, 2014b; Tóbiás et al. 2019).

\subsection{Electronic Structure and Spectroscopy of $\mathrm{CaOH}$}

The calcium monohydroxide radical is an open-shell system with a relatively complex electronic structure. To date, only the lowest-lying eight electronic states up to the $\tilde{G}^{2} \Pi$ state (Hailey et al. 1997) at approximately $32,633 \mathrm{~cm}^{-1}$ are known. In this work, we consider the lowest five electronic states $\left(\tilde{X}^{2} \Sigma^{+}\right.$, $\tilde{A}^{2} \Pi, \tilde{B}^{2} \Sigma^{+}, \tilde{C}^{2} \Delta$, and $\tilde{D}^{2} \Sigma^{+}$) and the transitions linking them, as shown in Figure 1. This choice was governed by the availability of laboratory rovibronic transition data in the literature. The wavenumber regions considered for each state were: $0-2599 \mathrm{~cm}^{-1}\left(\tilde{X}^{2} \Sigma^{+}\right) ; 15,966-17,677 \mathrm{~cm}^{-1}\left(\tilde{A}^{2} \Pi\right)$; $18,023-18,849 \mathrm{~cm}^{-1}\left(\tilde{B}^{2} \Sigma^{+}\right) ; 22,197-23,457 \mathrm{~cm}^{-1}\left(\tilde{C}^{2} \Delta\right)$; and $28,157-28,898 \mathrm{~cm}^{-1}\left(\tilde{D}^{2} \Sigma^{+}\right)$.

Interestingly, the spectrum of $\mathrm{CaOH}$ is affected by the Renner-Teller effect (see e.g., Jungen 2019 for a recent review), which is caused by the interaction of electronic orbital and vibrational angular momenta in linear molecules. This effect manifests itself when the molecule bends by lifting the degeneracy of the electronic states-for example, the first excited $\tilde{A}^{2} \Pi$ state of $\mathrm{CaOH}$ is split into two components, $A^{\prime}$ and $A^{\prime \prime}$, at bent configurations. The Renner-Teller effect complicates the spectral analysis due to the increased energy level congestion in band systems. But its treatment is necessary for a correct description and several experimental studies have considered Renner splittings in $\mathrm{CaOH}$ (Hilborn et al. 1983; Coxon et al. 1991; Jarman \& Bernath 1992; Li \& Coxon 1992, 1995).

\subsection{Vibronic Coupling, Symmetry, and Quantum Numbers}

MARVEL requires that each transition is between energy levels with unique state labels and quantum numbers that are consistent across the entire data set. For $\mathrm{CaOH}$, different authors have used different combinations of quantum numbers, as listed in Table 1. In this work, we have selected eight quantum numbers, shown in Table 2, that form a consistent set and allow each state to be uniquely labeled. The electronic state and the vibronic state are labeled along with the rotational angular momentum quantum number, $J$; the rotationless parity, 
Table 1

State Labels and Quantum Numbers Used by Different Authors for Energy Levels of $\mathrm{CaOH}$

\begin{tabular}{ll}
\hline \hline Label & Description \\
\hline Electronic state & Electronic state, e.g., $\tilde{X}^{2} \Sigma^{+}$ \\
Vibronic state & Vibronic state, e.g., $\tilde{X}^{2} \Delta, \mu \tilde{A}^{2} \Pi, \kappa \tilde{A}^{2} \Pi$ \\
$J$ & Rotational angular momentum \\
$e / f$ & Total (rotationless Kronig) "parity" \\
$c / d$ & Mulliken's label \\
$v_{1}$ & Symmetric stretching mode \\
$v_{2}$ & Bending mode (linear molecules) \\
$l$ & Vibrational angular momentum associated with $\nu_{2}$ mode \\
$v_{3}$ & Antisymmetric stretching mode \\
$\mu$ and $\kappa$ & Two vibronic components of $\tilde{A}{ }^{2} \Pi$ \\
$F_{1} / F_{2}$ & Spin components $J=N+1 / 2$ or $J=N-1 / 2$ \\
$\Omega$ & Projection of the total angular momentum on the $z$-axis.
\end{tabular}

Table 2

State Labels and Quantum Numbers of $\mathrm{CaOH}$ Adopted for the MARVEL Data Set

\begin{tabular}{ll}
\hline \hline Label & Description \\
\hline Vibronic state & Vibronic state, e.g., muA2Sigma $\left(\mu \tilde{A}^{2} \Sigma^{+}\right)$or X2Pi $\left(\tilde{X}^{2} \Pi\right)$ \\
$J$ & Rotational angular momentum \\
$e / f$ & Rotationless parity \\
$v_{1}$ & Symmetric stretching mode \\
$v_{2}$ & Bending mode (linear molecules) \\
$L$ & Vibrational angular momentum associated with $\nu_{2}$ \\
& $\quad$ mode $(L=|l|)$ \\
$v_{3}$ & Antisymmetric stretching mode \\
$F_{1} / F_{2}$ & Spin components $J=N+1 / 2$ or $J=N-1 / 2$ \\
\hline
\end{tabular}

$e / f ;$ and the quantum labels $F_{1}$ and $F_{2}$, denoting spin components $J=N+1 / 2$ and $J=N-1 / 2$, respectively. Normal mode notation $\left(v_{1}, v_{2}^{L}\right.$, and $\left.v_{3}\right)$ is used for the vibrational states, where $v_{1}, v_{2}$, and $v_{3}$ represent the symmetric stretch, bending, and asymmetric stretch modes. The quantum number, $L$, will be used to refer to the absolute value of the vibrational angular momentum quantum number, $l$, associated with the $\nu_{2}$ bending mode, $L=|l|$, where the vibrational quantum number, $l$, takes the following values:

$$
|l|=v_{2}, v_{2}-2, v_{2}-4, \ldots, 0(\text { or } 1)
$$

For example, the $v_{2}=1$ state has two degenerate components, $l= \pm 1$. The $v_{2}=2$ state assumes three states of $l= \pm 2$ and $l=0$. The $v_{2}=3$ state splits into four components, $l= \pm 3$ and $l= \pm 1$ and so on. The typical designation of the linear bending quantum mode therefore includes $|l|:\left(v_{1}, v_{2}^{|l|}\right.$, and $\left.v_{3}\right)$. For $v_{2}=0$ and often for $v_{2}=1$, the superscript $|l|$ can be omitted. Coupling with other degrees of freedom (such as molecular rotation) lifts the degeneracy of the $l \neq 0$ states. $^{3}$

When the molecule is linear, the $\mathcal{C}_{\infty \mathrm{v}}(M)$ molecular symmetry group is used to classify the rotation, vibration, and electronic degrees of freedom. The lowest five electronic states are $\tilde{X}^{2} \Sigma^{+}, \tilde{A}^{2} \Pi, \tilde{B}^{2} \Sigma^{+}, \tilde{C}^{2} \Delta$, and $\tilde{D}^{2} \Sigma^{+}$with the

\footnotetext{
3 Here, we use the linear triatomic version of the bending quantum number, $v_{2}$, which is related to the bent molecule case as $v_{2} \equiv v_{2}^{\text {linear }}=2 v_{2}^{\text {bent }}+L$, where $L$ is constrained to $L=K_{a} \pm \Lambda$ (Bunker \& Jensen 1998), and $K_{a}=\left|k_{a}\right|$ is the rotational quantum number associated with the projection of the total rotational angular momentum on the $a$-axis (or $z$-axis in our case).
}

projections of the electronic angular momentum on the $z$-axis $\Lambda=0(\tilde{X}, \tilde{B}$, and $\tilde{D}), \pm 1(\tilde{A})$, and $\pm 2(\tilde{C})$. The symmetry of the vibrational motion is controlled by the vibrational angular momentum quantum number, $L$, in the same way that the symmetry of the electronic state is defined by $\Lambda: \Sigma, \Pi, \Delta, \ldots$, for $l=0, \pm 1, \pm 2, \ldots$, respectively. The electronic angular momentum can be coupled to the vibrational angular momentum giving the vibronic angular momentum with projections $\Lambda+l$, which are also described using the notation $\Sigma^{ \pm}, \Pi, \Delta, \Phi, \ldots$. These vibronic symmetries are then used to label the final vibronic states while the electronic symmetries are omitted. Consider, for instance, the vibrational state $\left(0,1^{1}\right.$, $0)$ in the first excited electronic state $\tilde{A}^{2} \Pi$. Coupling its vibrational angular momentum with $l= \pm 1$ (П) and the electronic angular momentum with $\Lambda= \pm 1$ leads to the three vibronic components $|\Lambda+l|=0,0,2$, i.e., $\Sigma^{+}, \Sigma^{-}$, and $\Delta$, with a doubly degenerate state, $\Delta$. These vibronic states are then assigned $\tilde{A}\left(0,1^{1}, 0\right){ }^{2} \Sigma^{ \pm}$and $\tilde{A}\left(0,1^{1}, 0\right){ }^{2} \Delta$.

The bending motion of the molecule $\left(v_{2}>0\right)$ lifts the electronic degeneracy of $\Lambda \neq 0$ states $\left(\tilde{A}^{2} \Pi\right.$ and $\left.\tilde{C}^{2} \Delta\right)$ via the Renner-Teller interaction ( $\Lambda$ doubling; see, e.g., Pople 1960; Hougen 1962a, 1962b, 1962c). There are two alternative notations for these components used in the literature. Experimental studies of $\mathrm{CaOH}$ tend to use $\mu$ and $\kappa$ for the two vibronic sub-bands associated with the Renner-Teller splitting, while a more general consideration denotes the two RennerTeller components $A^{\prime}$ and $A^{\prime \prime}$ of the $C_{s}$ symmetry group to classify the molecular states, as well as the different degrees of freedom of $\mathrm{CaOH}$. According to this convention, the $\kappa$ levels are always higher in energy than the $\mu$ levels of the same $J$ (Hougen 1962c). In the above example of $\left(0,1^{1}, 0\right)$ in the excited electronic state, $\tilde{A}^{2} \Pi$, the final vibronic notations inherit the symmetry of a linear molecule and are given by $\tilde{A}\left(0,1^{1}, 0\right) \mu / \kappa^{2} \Sigma^{ \pm}$and $\tilde{A}\left(0,1^{1}, 0\right)^{2} \Delta$.

\subsection{Symmetry Considerations}

When coupling different angular momenta of $\mathrm{CaOH}$, it is common to first couple the electronic and vibrational angular momenta toward the vibronic momentum with projections $\Lambda+l$ (also assigned ${ }^{2} \Sigma^{ \pm}, \Pi, \Delta, \Phi, \ldots$ ) and then with the unpaired spin $(S=1 / 2)$ toward the total angular momentum with the projection $\Omega=\Lambda+l+\Sigma$, where $\Sigma$ is the projection of the electron spin angular momentum on the $z$-axis. ${ }^{4}$ For $\mathrm{CaOH}$, it is common to use the $F_{1}$ and $F_{2}$ quantum labels to distinguish the two spin components with $|\Lambda+l| \pm 1 / 2$. For example, in the ${ }^{2} \Sigma$ states, the rotational angular momentum $\boldsymbol{N}$ is coupled to spin as $\boldsymbol{J}=\boldsymbol{N}+\boldsymbol{S}$ and thus their projections on the $z$-axis (at linear configurations) are related by $J=N \pm 1 / 2$, where $N$ is the rotational angular momentum. Each rotational state of ${ }^{2} \Sigma$ consists of two spin subcomponents, assigned $F_{1}$ and $F_{2} . \Omega$ is sometimes used as an alternative to $F_{1}$ and $F_{2}$ to identify vibronic subcomponents, e.g., as ${ }^{2} \Pi_{1 / 2}$ and ${ }^{2} \Pi_{3 / 2},{ }^{2} \Delta_{3 / 2}$ and ${ }^{2} \Delta_{5 / 2}$, etc. Most of the sources presented in this work, however, use the $F_{1}$ and $F_{2}$ notation.

The symmetry of the rovibronic state is a product of the symmetries of the electronic, vibrational, spin, and rotational parts and can also be associated with the corresponding angular momenta $\boldsymbol{G}, \boldsymbol{L}, \boldsymbol{S}$, and $\boldsymbol{N}$. However, the final rovibronic state can only be $\Sigma^{+}$(parity is + ) or $\Sigma^{-}$(parity is - ) of the

\footnotetext{
$\overline{4}$ Note that this label $\Sigma$ is different from the label $\Sigma$ used to assign the $\Lambda=0$ state.
} 
molecular symmetry group $\mathcal{C}_{\infty \mathrm{v}}(M)$ (Watson 1970). The parity of ${ }^{2} \Sigma^{+}$or ${ }^{2} \Sigma^{-}$states are $(-1)^{N}$ and $(-1)^{N+1}$, respectively. The state parity always changes for the electric dipole transitions $+\leftrightarrow-$, which can be used to reconstruct the parity of the upper/lower state if only one of them is known. The rotationless $e / f$ parity is related to the $+/-$ parity as follows: levels with parity $+(-1)^{J-0.5}$ are $e$ levels, and levels with parity $-(-1)^{J-0.5}$ are $f$ levels (Brown et al. 1975). Some authors use the Mulliken' labels $c$ and $d$ (Mulliken 1931) to distinguish states of different parities - for example ,in the case of $v_{2}=3$ (Ziurys et al. 1996) with $\left(0,3^{1 c}, 0\right),\left(0,3^{1 d}, 0\right)$, $\left(0,3^{3 c}, 0\right)$, and $\left(0,3^{3 d}, 0\right)$.

The electric dipole selection rules are

$$
\begin{gathered}
J^{\prime}=J^{\prime \prime} \pm 1, \\
e \leftrightarrow e \quad \text { and } f \leftrightarrow f \quad \Delta J= \pm 1, \\
e \leftrightarrow f \quad \Delta J=0, \\
\Sigma^{+} \leftrightarrow \Sigma^{-} \quad \text { (total symmetry). }
\end{gathered}
$$

\subsection{Examples of Coupling Vibration, Spin, and Electronic Angular Momenta}

For non- $\Sigma$ states, the interaction with the electron spin can involve the vibrational and electronic angular momentum. For example, the vibrational excitation $\left(0,1^{1}, 0\right)$ of the electronic state $\tilde{X}^{2} \Sigma^{+}$gives rise to the total vibronic angular momentum of the symmetry ${ }^{2} \Pi(l= \pm 1)$, referenced to as $\tilde{X}\left(0,1^{1}, 0\right)^{2} \Pi$. The resulting rovibronic energy pattern forms a quartet, which can be designated using the four combinations of $e$ and $f$, and $F_{1}$ and $F_{2}$.

Including the electronic angular momentum complicates this picture even more. For example, in order to couple all angular momenta in the vibronic state $\tilde{A}\left(0,1^{1}, 0\right)^{2} \Pi$, in this work, we first couple the electronic and vibrational momenta with projections $l= \pm 1$ and $\Lambda= \pm 1$, respectively, toward the vibronic angular momentum with $l+\Lambda=0^{+/-}, \pm 2$. The zero vibronic components $0^{+/-}$have two symmetries, ${ }^{2} \Sigma^{+}$and ${ }^{2} \Sigma^{-}$, with $|\Omega|=1 / 2$. In this case, $F_{1}$ and $F_{2}$ are redundant and can be chosen to match $e$ and $f$ states. The state with $l+\Lambda= \pm 2$ has the symmetry ${ }^{2} \Delta$ with $|\Omega|$ taking values $3 / 2$ and $5 / 2$ and therefore can also be designated $\left(0,1^{1}, 0\right)^{2} \Delta_{3 / 2}\left(F_{1}\right)$ and $\left(0,1^{1}, 0\right)^{2} \Delta_{5 / 2}\left(F_{2}\right)$, respectively, where the labels $F_{1}$ and $F_{2}$ are assigned to the $|\Omega|=1 / 2$ and $3 / 2$ states based on the order of the corresponding energy values.

Consider another example of the $\tilde{A}(0,2,0)^{2} \Pi$ state $(\Lambda= \pm 2$ and $l=0, \pm 2$ ) with the vibronic angular momenta $\Lambda+l= \pm 1$ and \pm 3 , which represent the vibronic states ${ }^{2} \Pi$ and ${ }^{2} \Phi$, respectively. The $\Lambda= \pm 2$ degeneracies are lifted due to the interaction with the bending mode (Renner-Teller) leading to the $\mu$ and $\kappa$ subcomponents (or $A^{\prime}$ and $A^{\prime \prime}$ ). The interaction with spin introduces the spin splitting with the final values of the projections of the angular momenta $1 / 2$ and $3 / 2$ $\left({ }^{2} \Pi\right)$ or $5 / 2$ and $7 / 2\left({ }^{2} \Phi\right)$, i.e., to ${ }^{2} \Pi_{1 / 2},{ }^{2} \Pi_{3 / 2},{ }^{2} \Phi_{5 / 2}$, and ${ }^{2} \Phi_{7 / 2}$. However, if $l=0$ but $\Lambda \neq 0$, such as e.g., for the $\tilde{A}(0,0,0)^{2} \Pi$ state, the final projections of the total angular momentum $\Omega=\Lambda+\Sigma$ are $\pm 1 / 2$ and $\pm 3 / 2$, and these states can be assigned ${ }^{2} \Pi_{1 / 2}$ and ${ }^{2} \Pi_{3 / 2}$, respectively.

\section{Experimental Data Sources}

Spectroscopic data was extracted from 13 published sources (Hilborn et al. 1983; Bernath \& Kinsey-Nielsen 1984; Bernath \& Brazier 1985; Coxon et al. 1991, 1992, 1994; Jarman \& Bernath 1992; Li \& Coxon 1992, 1995; Ziurys et al. 1992, 1996;
Scurlock et al. 1993; Dick et al. 2006). These data are summarized in Table 3. Only data from Dick et al. (2006) was provided in digital format while all other literature sources had to be processed using digitization software. A unique reference label was assigned to each extracted transition, which is a requirement for the MARVEL input file. The reference indicates the data source, table (or page) and line number that the transition originates from. The data source tag (for example, 06DiShWa) is based on the notation employed by the International Union of Pure and Applied Chemistry (IUPAC) task group on water (Tennyson et al. 2010, 2014a).

Aside from the numerous experimental measurements of $\mathrm{CaOH}$ spectra, there have been several studies investigating the electronic structure of $\mathrm{CaOH}$ using quantum chemical methods (Bauschlicher \& Partridge 1984; Bauschlicher et al. 1986, 1990; Ortiz 1990; Kong \& Boyd 1996; Koput \& Peterson 2002; Theodorakopoulos et al. 2002; Taylor et al. 2005) but these have largely focused on molecular structures and properties rather than rovibronic spectroscopy.

\subsection{Comments on Literature Sources}

83HiQiHa: Hilborn et al. (1983) contains transitions from the $\tilde{A}(0,0,0)^{2} \Pi-\tilde{X}(0,0,0)^{2} \Sigma^{+}$band. Most of these data are included in the more recent study by Dick et al. (2006) but some of the line positions show deviations up to $0.3 \mathrm{~cm}^{-1}$. For example, in the transition $\tilde{A}(0,0,0)^{2} \Pi, J=36.5 F=2$, $e \leftarrow \tilde{X}(0,0,0)^{2} \Pi$, and $J=36.5 F=2, f$ appears in Hilborn et al. (1983) as $16,029.986 \mathrm{~cm}^{-1}$ and in Dick et al. (2006) as $16,030.285 \mathrm{~cm}^{-1}$. We believe that the recent data (Dick et al. 2006) is more reliable. In these instances, we have removed the lines by Hilborn et al. (1983) from our MARVEL analysis by making the corresponding frequency value negative (MARVEL convention).

84BeKi: Bernath \& Kinsey-Nielsen (1984) contains transitions from the $\tilde{B}(0,0,0)^{2} \Sigma^{+}-\tilde{X}(0,0,0)^{2} \Sigma^{+}$and $\tilde{B}(1,0,0)^{2} \Sigma^{+}-\tilde{X}(1,0,0)^{2} \Sigma^{+}$band.

85BeBr: Bernath \& Brazier (1985) contains transitions from the $\tilde{A}(0,0,0)^{2} \Pi-\tilde{X}(0,0,0)^{2} \Sigma^{+}$band.

91CoLiPr: Coxon et al. (1991) contains transitions from the $\tilde{A}(1,0,0)^{2} \Pi-\tilde{X}(0,0,0)^{2} \Sigma^{+}$band.

92LiCo: Li \& Coxon (1992) reported transitions from the $\tilde{A}\left(0,2^{0}, 0\right)^{2} \Pi-\tilde{X}(0,0,0)^{2} \Sigma^{+}$band system.

92CoLiPr: Coxon et al. (1992) studied the $\tilde{A}^{2} \Pi-$ $\tilde{X}^{2} \Sigma^{+}$system, covering the $\tilde{A}(1,0,0)^{2} \Pi-\tilde{X}(1,0,0)^{2} \Sigma^{+}$, $\tilde{A}(1,0,0)^{2} \Pi-\tilde{X}(2,0,0)^{2} \Sigma^{+}, \quad \tilde{A}(1,0,0)^{2} \Pi-\tilde{X}(3,0,0)^{2} \Sigma^{+}$, $\tilde{A}(1,0,0)^{2} \Pi-\tilde{X}(4,0,0)^{2} \Sigma^{+}, \quad \tilde{A}(1,0,0)^{2} \Pi-, \tilde{X}\left(0,2^{0}, 0\right)^{2} \Sigma^{+}$ and $\tilde{A}(1,0,0)^{2} \Pi-\tilde{X}\left(0,2^{2}, 0\right)^{2} \Delta$ vibronic bands.

92ZiBaAn: Ziurys et al. (1992) investigated 11 pure rotational transitions in the $\tilde{X}(0,0,0)^{2} \Sigma^{+}$ground state. The total angular momentum quantum number, $F$, used to account for hyperfine structure was not included in our data set. Instead, the uncertainty of these transitions was increased to match the hyperfine splittings. The data set was converted to $\mathrm{cm}^{-1}$ from the original units of $\mathrm{MHz}$.

92JaBe: Jarman \& Bernath (1992) studied the $\tilde{C}{ }^{2} \Delta-\tilde{X}^{2} \Sigma^{+}$ system covering the $\tilde{C}\left(0,1^{1}, 0\right)^{2} \Pi-\tilde{X}(0,0,0)^{2} \Sigma^{+}$band.

93ScFlSt: Scurlock et al. (1993) investigated the hyperfine structure of the three lowest pure rotational transitions of the $\tilde{X}(0,0,0)^{2} \Sigma^{+}$state. The total angular momentum quantum number, $F$, used to account for hyperfine structure was not included in our data set. Instead, the uncertainties for these 
Table 3

Experimental Sources of $\mathrm{CaOH}$ Spectra and Their Coverage

\begin{tabular}{|c|c|c|c|c|c|c|}
\hline Experimental Source & Electron. States & $\left(v_{1} v_{2} v_{3}\right)^{\mathrm{a}}$ & $J$ Range & Coverage $\left(\mathrm{cm}^{-1}\right)$ & $A / V^{b}$ & $\Delta\left(\mathrm{cm}^{-1}\right)^{\mathrm{c}}$ \\
\hline 83HiQiHa (Hilborn et al. 1983) & $\tilde{A}^{2} \Pi-\tilde{X}^{2} \Sigma^{+}$ & $(000)-(000)$ & $1.5-47.5$ & $15,928-16,045$ & $131 / 115$ & 0.3 \\
\hline $84 \mathrm{BeKi}$ & $\tilde{B}^{2} \Sigma^{+}-\tilde{X}^{2} \Sigma^{+}$ & $(000)-(000)$ & $1.5-26.5$ & $18,008-18,036$ & $73 / 73$ & 0.01 \\
\hline (Bernath \& Kinsey-Nielsen 1984) & $\tilde{B}^{2} \Sigma^{+}-\tilde{X}^{2} \Sigma^{+}$ & $(100)-(100)$ & $5.5-25.5$ & $18,007-18,033$ & $48 / 48$ & 0.01 \\
\hline 85BeBr (Bernath \& Brazier 1985) & $\tilde{A}^{2} \Pi-\tilde{X}^{2} \Sigma^{+}$ & $(000)-(000)$ & $1.5-53.5$ & $15,970-16,068$ & $155 / 155$ & 0.01 \\
\hline 91CoLiPr (Coxon et al. 1991) & $\tilde{A}^{2} \Pi-\tilde{X}^{2} \Sigma^{+}$ & $(100)-(000)$ & $0.5-56.5$ & $16,578-16,659$ & $201 / 201$ & 0.005 \\
\hline 92LiCo (Li \& Coxon 1992) & $\tilde{A}^{2} \Pi-\tilde{X}^{2} \Sigma^{+}$ & $(020)-(000)$ & $1.5-40.5$ & $16,641-16,807$ & $322 / 322$ & 0.01 \\
\hline \multirow[t]{6}{*}{ 92CoLiPr (Coxon et al. 1992) } & $\tilde{A}^{2} \Pi-\tilde{X}^{2} \Sigma^{+}$ & $(100)-\left(02^{0} 0\right)$ & $4.5-29.5$ & $15,951-15,988$ & $25 / 25$ & 0.104 \\
\hline & $\tilde{A}^{2} \Pi-\tilde{X}^{2} \Sigma^{+}$ & $(100)-\left(02^{2} 0\right)$ & $4.5-26.5$ & $15,909-15,960$ & $48 / 48$ & 0.056 \\
\hline & $\tilde{A}^{2} \Pi-\tilde{X}^{2} \Sigma^{+}$ & $(100)-(100)$ & $5.5-29.5$ & $15,957-16,069$ & $54 / 54$ & 0.064 \\
\hline & $\tilde{A}^{2} \Pi-\tilde{X}^{2} \Sigma^{+}$ & $(100)-(200)$ & $4.5-29.5$ & $15,352-15,392$ & $42 / 42$ & 0.056 \\
\hline & $\tilde{A}^{2} \Pi-\tilde{X}^{2} \Sigma^{+}$ & $(100)-(300)$ & $4.5-27.5$ & $14,762-14,798$ & $30 / 30$ & 0.17 \\
\hline & $\tilde{A}^{2} \Pi-\tilde{X}^{2} \Sigma^{+}$ & $(100)-(400)$ & $14.5-25.5$ & $14,206-14,214$ & $9 / 9$ & 0.10 \\
\hline 92ZiBaAn (Ziurys et al. 1992) & $\tilde{X}^{2} \Sigma^{+}-\tilde{X}^{2} \Sigma^{+}$ & $(000)-(000)$ & $2.5-16.5$ & $2.67-10.69$ & $44 / 44$ & $1.7 \times 10^{-6}$ \\
\hline \multirow[t]{2}{*}{ 92JaBe (Jarman \& Bernath 1992) } & $\tilde{X}^{2} \Sigma^{+}-\tilde{X}^{2} \Sigma^{+}$ & $(000)-(000)$ & $2.5-38.5$ & $0.045-10.69$ & $25 / 25$ & $1 \times 10^{-5}$ \\
\hline & $\tilde{C}^{2} \Delta-\tilde{X}^{2} \Sigma^{+}$ & $(010)-(000)$ & $0.5-62.5$ & $0.045-22,247$ & $373 / 374$ & 0.33 \\
\hline \multirow[t]{2}{*}{ 94CoLiPr (Coxon et al. 1994) } & $\tilde{A}^{2} \Pi-\tilde{X}^{2} \Sigma^{+}$ & $\left(01^{1} 0\right)-\left(01^{1} 0\right)$ & $1.5-38.5$ & $15,923-16,095$ & $409 / 409$ & 0.06 \\
\hline & $\tilde{A}^{2} \Pi-\tilde{X}^{2} \Sigma^{+}$ & $\left(01^{1} 0\right)-(000)$ & $0.5-41.5$ & $16,289-16,461$ & $409 / 409$ & 0.016 \\
\hline 93ScFlSt (Scurlock et al. 1993) & $\tilde{X}^{2} \Sigma^{+}-\tilde{X}^{2} \Sigma^{+}$ & $(000)-(000)$ & $0.5-3.5$ & $0.67-2.01$ & $26 / 26$ & $3.3 \times 10^{-7}$ \\
\hline \multirow[t]{2}{*}{ 95LiCo (Li \& Coxon 1995) } & $\tilde{A}^{2} \Pi-\tilde{X}^{2} \Sigma^{+}$ & $\left(01^{1} 0\right)-\left(01^{1} 0\right)$ & $1.5-61.5$ & $15,935-16,428$ & $640 / 640$ & 0.01 \\
\hline & $\tilde{A}^{2} \Pi-\tilde{X}^{2} \Sigma^{+}$ & $\left(01^{1} 0\right)-(000)$ & $40.5-60.5$ & $15,935-16,428$ & $640 / 640$ & 0.01 \\
\hline \multirow[t]{5}{*}{ 96ZiFlAn (Ziurys et al. 1996) } & $\tilde{X}^{2} \Sigma^{+}-\tilde{X}^{2} \Sigma^{+}$ & $(000)-(000)$ & $2.5-19.5$ & $2.67-12.69$ & $28 / 28$ & $5 \times 10^{-6}$ \\
\hline & $\tilde{X}^{2} \Sigma^{+}-\tilde{X}^{2} \Sigma^{+}$ & $\left(01^{1} 0\right)-\left(01^{1} 0\right)$ & $12.5-19.5$ & $9.32-12.64$ & $23 / 23$ & $5 \times 10^{-6}$ \\
\hline & $\tilde{X}^{2} \Sigma^{+}-\tilde{X}^{2} \Sigma^{+}$ & $\left(02^{0} 0\right)-\left(02^{0} 0\right)$ & $12.5-19.5$ & $9.31-12.64$ & $36 / 36$ & $8.3 \times 10^{-6}$ \\
\hline & $\tilde{X}^{2} \Sigma^{+}-\tilde{X}^{2} \Sigma^{+}$ & $\left(02^{2} 0\right)-\left(02^{2} 0\right)$ & $12.5-19.5$ & $9.31-12.64$ & $36 / 36$ & $8.3 \times 10^{-6}$ \\
\hline & $\tilde{X}^{2} \Sigma^{+}-\tilde{X}^{2} \Sigma^{+}$ & $(100)-(100)$ & $12.5-19.5$ & $9.29-12.61$ & $12 / 12$ & $8.3 \times 10^{-6}$ \\
\hline \multirow[t]{2}{*}{ 06DiShWa (Dick et al. 2006) } & $\tilde{A}^{2} \Pi-\tilde{X}^{2} \Sigma^{+}$ & $(000)-(000)$ & $1.5-53.5$ & $15,928-16,068$ & $267 / 267$ & 0.017 \\
\hline & $\tilde{D}^{2} \Sigma^{+}-\tilde{A}^{2} \Pi$ & $(000)-(000)$ & $0.5-45.5$ & $12,108-12,232$ & $206 / 206$ & 0.018 \\
\hline
\end{tabular}

Notes.

${ }^{a}$ Vibrational quantum numbers for upper and lower states (see the text).

b Available/verified number of lines from the literature source.

${ }^{c}$ Average uncertainty of transition wavenumber data.

transitions were increased to $0.0001 \mathrm{~cm}^{-1}$ to account for the fact that we have neglected hyperfine effects.

94CoLiPr: Coxon et al. (1994) measured transitions in the $\tilde{A}^{2} \Pi-\tilde{X}^{2} \Sigma^{+}$band system: $\tilde{A}\left(0,1^{1}, 0\right)^{2} \Sigma^{+}-\tilde{X}\left(0,1^{1}, 0\right)^{2} \Pi$ and $\tilde{A}\left(0,1^{1}, 0\right)^{2} \Sigma^{-}-\tilde{X}(0,0,0)^{2} \Sigma^{+}$bands. The upper doubling states are assigned $\kappa^{2} \Sigma^{-}$or $\mu^{2} \Sigma^{+}$.

95LiCo: $\mathrm{Li} \&$ Coxon (1995) studied the $v_{2}=1$ bending vibrational levels of the $\tilde{A}^{2} \Pi$ and $\tilde{X}^{2} \Sigma^{+}$states, covering transitions of the following bands: $\tilde{A}\left(0,1^{1}, 0\right) \kappa^{2} \Sigma^{-}$, ${ }^{2} \Delta, \mu^{2} \Sigma^{+} \leftarrow \tilde{X}\left(0,1^{1}, 0\right)^{2} \Pi$, and $(0,0,0)^{2} \Sigma^{+}$. It should be noted that $\tilde{A}\left(0,1^{1}, 0\right)-\tilde{X}(0,0,0)$ is electric dipole forbidden. Even though the $\tilde{A}(0,1,0) \mu^{2} \Sigma^{+}$and $\tilde{A}\left(0,1^{1}, 0\right) \kappa^{2} \Sigma^{-}$states can be correlated to $A^{\prime}$ and $A^{\prime \prime}$, we retained the experimental labels $\mu$ and $\kappa$. The $e / f$ parity of all states was reconstructed from the $+/-$ parities $p$, which in turn were obtained from the parity $p^{\prime \prime}$ of the lower vibronic state $\Sigma^{+}$as $(-1)^{N}$. For non- $\Sigma$ lower vibronic states $\left(\tilde{X}^{2} \Pi\right)$, we used the upper state parity instead if the vibronic symmetries were either $\Sigma^{+}(\mu)$ or $\Sigma^{-}(\kappa)$ : the parities $p^{\prime}$ are $(-1)^{N^{\prime}}$ or $-(-1)^{N^{\prime}}$, respectively.

96ZiFlAn: Ziurys et al. (1996) has pure rotational and rovibrational transitions in the $\tilde{X}^{2} \Sigma^{+}$ground electronic state: $(0,0,0),\left(0,1^{1 c}, 0\right),\left(0,1^{1 d}, 0\right),\left(0,2^{0}, 0\right),\left(0,2^{2 c}, 0\right)$, and $(0$, $\left.2^{2 d}, 0\right)$ where the superscript $c / d$ represents $l$-type doubling effects. The $\left(0,1^{1}, 0\right)$ and $\left(0,2^{2}, 0\right)$ vibronic states of $\tilde{X}^{2} \Sigma^{+}$ have the vibrational symmetries of ${ }^{2} \Pi$ and ${ }^{2} \Delta$, respectively. The correlation between $c / d$ and $e / f$ does not appear to follow the rules given by Brown et al. (1975). In order to reconstruct this correlation, we used the $\left(0,1^{1}, 0\right)$ spectroscopic constants from Ziurys et al. (1996) in the program PGOPHER (Western 2017), assuming the vibronic symmetry ${ }^{2} \Pi$, and computed the corresponding transitions allowing us to correlate the $e / f$ and $c / d$ parities as follows: for $c, F_{1}=e$ and $F_{2}=f$; for $d, F_{1}=f$ and $F_{2}=e$, but only for the $(0,0,0),\left(0,1^{1 c}, 0\right)$, and $\left(0,1^{1 d}, 0\right)$ states. The $\mathrm{CaOH}$ spectroscopic constants of $\tilde{X}\left(0,2^{2}, 0\right)^{2} \Delta$ by Ziurys et al. (1996) only work with PGOPHER if assuming the symmetry ${ }^{2} \Pi$ instead of ${ }^{2} \Delta$, which, in this case, worked with the same conversion rules as for the $\left(0,1^{1}, 0\right)$ transitions. Apparently, this is due to the effective rotational Hamiltonian model used in the fit in the original work. The data set was converted to $\mathrm{cm}^{-1}$ from the original units of $\mathrm{MHz}$.

O6DiShWa: Dick et al. (2006) covers transitions from the $\tilde{A}(0,0,0)^{2} \Pi-\tilde{X}(0,0,0)^{2} \Sigma^{+}$and $\tilde{D}(0,0,0)^{2} \Sigma^{+}-$ $\tilde{A}(0,0,0)^{2} \Pi$ bands. In their analysis, Dick et al. (2006) used the $\tilde{X}(0,0,0)^{2} \Sigma^{+}-\tilde{X}(0,0,0)^{2} \Sigma^{+}$transitions from the work by Ziurys et al. (1992). A number of the $\tilde{A}-\tilde{X}$ transitions were measured in the work of Hilborn et al. (1983).

\section{Results and Discussion}

Our final MARVEL transition file (the input, see Table 4) consists of 3204 experimental (excluding the 16 transitions from Hilborn et al. 1983 deemed less reliable) and 20 pseudo- 
Table 4

Extract from the MARVEL Transition File

\begin{tabular}{|c|c|c|c|c|c|c|c|c|c|c|c|c|c|c|c|c|c|c|}
\hline \multirow{2}{*}{$\tilde{\nu}$} & \multirow{2}{*}{ unc., $\mathrm{cm}^{-1}$} & \multicolumn{8}{|c|}{ Quantum "Numbers" of Upper States } & \multicolumn{8}{|c|}{ Quantum "Numbers" of Lower States } & \multirow{2}{*}{ Source } \\
\hline & & Vibronic $^{\prime}$ & $J^{\prime}$ & $e^{\prime} / f^{\prime}$ & $v_{1}^{\prime}$ & $v_{2}^{\prime}$ & $L^{\prime}$ & $v_{3}^{\prime}$ & $F_{1}^{\prime} / F_{2}^{\prime}$ & Vibronic" $^{\prime \prime}$ & $J^{\prime \prime}$ & $e^{\prime \prime} / f^{\prime \prime}$ & $v_{1}^{\prime \prime}$ & $v_{2}^{\prime \prime}$ & $L^{\prime \prime}$ & $v_{3}^{\prime \prime}$ & $F_{1}^{\prime \prime} / F_{2}^{\prime \prime}$ & \\
\hline 16026.295 & 0.005 & $\mathrm{~A} 2 \mathrm{Pi}$ & 3.5 & $f$ & 0 & 0 & 0 & 0 & F2 & $\mathrm{X} 2$ Sigma + & 4.5 & $f$ & 0 & 0 & 0 & 0 & $\mathrm{~F} 2$ & 06DiShWa1 \\
\hline 16025.364 & 0.01 & $\mathrm{~A} 2 \mathrm{Pi}$ & 4.5 & $f$ & 0 & 0 & 0 & 0 & $\mathrm{~F} 2$ & $\mathrm{X} 2$ Sigma + & 5.5 & $f$ & 0 & 0 & 0 & 0 & $\mathrm{~F} 2$ & 06DiShWa2 \\
\hline 16024.464 & 0.005 & $\mathrm{~A} 2 \mathrm{Pi}$ & 5.5 & $f$ & 0 & 0 & 0 & 0 & $\mathrm{~F} 2$ & $\mathrm{X} 2$ Sigma + & 6.5 & $f$ & 0 & 0 & 0 & 0 & $\mathrm{~F} 2$ & 06DiShWa3 \\
\hline 16023.571 & 0.005 & $\mathrm{~A} 2 \mathrm{Pi}$ & 6.5 & $f$ & 0 & 0 & 0 & 0 & F2 & $\mathrm{X} 2$ Sigma + & 7.5 & $f$ & 0 & 0 & 0 & 0 & $\mathrm{~F} 2$ & 06DiShWa4 \\
\hline 16022.696 & 0.01 & $\mathrm{~A} 2 \mathrm{Pi}$ & 7.5 & $f$ & 0 & 0 & 0 & 0 & F2 & $\mathrm{X} 2$ Sigma + & 8.5 & $f$ & 0 & 0 & 0 & 0 & $\mathrm{~F} 2$ & 06DiShWa5 \\
\hline 16021.842 & 0.005 & $\mathrm{~A} 2 \mathrm{Pi}$ & 8.5 & $f$ & 0 & 0 & 0 & 0 & F2 & $\mathrm{X} 2$ Sigma + & 9.5 & $f$ & 0 & 0 & 0 & 0 & $\mathrm{~F} 2$ & 06DiShWa6 \\
\hline 16021.002 & 0.005 & $\mathrm{~A} 2 \mathrm{Pi}$ & 9.5 & $f$ & 0 & 0 & 0 & 0 & F2 & $\mathrm{X} 2$ Sigma + & 10.5 & $f$ & 0 & 0 & 0 & 0 & $\mathrm{~F} 2$ & 06DiShWa7 \\
\hline 16020.18 & 0.005 & $\mathrm{~A} 2 \mathrm{Pi}$ & 10.5 & $f$ & 0 & 0 & 0 & 0 & F2 & $\mathrm{X} 2$ Sigma + & 11.5 & $f$ & 0 & 0 & 0 & 0 & $\mathrm{~F} 2$ & 06DiShWa8 \\
\hline 16019.375 & 0.005 & $\mathrm{~A} 2 \mathrm{Pi}$ & 11.5 & $f$ & 0 & 0 & 0 & 0 & F2 & $\mathrm{X} 2$ Sigma + & 12.5 & $f$ & 0 & 0 & 0 & 0 & $\mathrm{~F} 2$ & 06DiShWa9 \\
\hline 16018.586 & 0.005 & $\mathrm{~A} 2 \mathrm{Pi}$ & 12.5 & $f$ & 0 & 0 & 0 & 0 & F2 & $\mathrm{X} 2$ Sigma + & 13.5 & $f$ & 0 & 0 & 0 & 0 & $\mathrm{~F} 2$ & 06DiShWa10 \\
\hline 16017.815 & 0.005 & $\mathrm{~A} 2 \mathrm{Pi}$ & 13.5 & $f$ & 0 & 0 & 0 & 0 & F2 & $\mathrm{X} 2$ Sigma + & 14.5 & $f$ & 0 & 0 & 0 & 0 & $\mathrm{~F} 2$ & 06DiShWa11 \\
\hline
\end{tabular}

Note. The quantum numbers/labels are described in Table 2. The MARVEL frequency wavenumber, $\tilde{\nu}$, and uncertainties are in $\mathrm{cm}^{-1}$.

(This table is available in its entirety in FITS format.) 
Table 5

Extract from the MARVEL Energy File

\begin{tabular}{|c|c|c|c|c|c|c|c|c|c|c|}
\hline \multirow[b]{2}{*}{ Vibronic } & \multicolumn{7}{|c|}{ Quantum "Numbers" } & \multirow{2}{*}{$\tilde{E} \quad \mathrm{~cm}^{-1}$} & \multirow{2}{*}{$\begin{array}{l}\text { unc. } \\
\qquad \mathrm{cm}^{-1}\end{array}$} & \multirow{2}{*}{$\begin{array}{l}\text { No. of } \\
\text { Trans. }\end{array}$} \\
\hline & $J$ & $e / f$ & $v_{1}$ & $v_{2}$ & $L$ & $v_{3}$ & $F_{1} / F_{2}$ & & & \\
\hline X2Delta & 24.5 & $e$ & 0 & 2 & 2 & 0 & $\mathrm{~F} 1$ & 911.045055 & 0.05 & 1 \\
\hline X2Delta & 24.5 & $f$ & 0 & 2 & 2 & 0 & $\mathrm{~F} 2$ & 927.860974 & 0.05 & 1 \\
\hline X2Delta & 25.5 & $f$ & 0 & 2 & 2 & 0 & $\mathrm{~F} 2$ & 944.898055 & 0.05 & 1 \\
\hline X2Delta & 26.5 & $f$ & 0 & 2 & 2 & 0 & $\mathrm{~F} 2$ & 963.000974 & 0.05 & 1 \\
\hline muA2Sigma & 0.5 & $e$ & 0 & 1 & 1 & 0 & $\mathrm{~F} 1$ & 16310.22824 & 0.005 & 1 \\
\hline muA2Sigma & 0.5 & $f$ & 0 & 1 & 1 & 0 & $\mathrm{~F} 2$ & 16310.70825 & 0.005 & 1 \\
\hline muA2Sigma & 1.5 & $e$ & 0 & 1 & 1 & 0 & $\mathrm{~F} 1$ & 16311.01157 & 0.007071 & 2 \\
\hline muA2Sigma & 1.5 & $f$ & 0 & 1 & 1 & 0 & $\mathrm{~F} 2$ & 16311.96407 & 0.070711 & 2 \\
\hline muA2Sigma & 2.5 & $e$ & 0 & 1 & 1 & 0 & $\mathrm{~F} 1$ & 16312.46245 & 0.003462 & 6 \\
\hline muA2Sigma & 2.5 & $f$ & 0 & 1 & 1 & 0 & $\mathrm{~F} 2$ & 16313.911 & 0.003333 & 3 \\
\hline muA2Sigma & 3.5 & $e$ & 0 & 1 & 1 & 0 & $\mathrm{~F} 1$ & 16314.6022 & 0.004994 & 2 \\
\hline
\end{tabular}

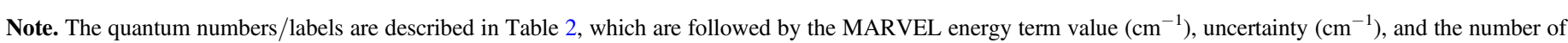
transitions supporting the state in question.

(This table is available in its entirety in FITS format.)

experimental (PGOPHER) transitions and has the following structure:

$$
\tilde{\nu} \text { unc } \mathrm{QN}^{\prime} \mathrm{QN}^{\prime \prime} \text { source }_{i}
$$

where $\tilde{\nu}$ is the transition wavenumber $\left(\mathrm{cm}^{-1}\right)$, unc is the experimental uncertainty $\left(\mathrm{cm}^{-1}\right), \mathrm{QN}^{\prime}$ and $\mathrm{QN}^{\prime \prime}$ are the quantum numbers of the upper and lower states, respectively, and the "source" is the abbreviation of the literature source concatenated with a counting number $i$ of the data from this source. source $_{i}$ is a unique ID of the transitions in the MARVEL data set. The pseudo-experimental transition wavenumbers were reconstructed with the PGOPHER program using the spectroscopic constants from Li \& Coxon (1995) and Ziurys et al. (1996) to support the corresponding data sets compensating for missing lines. Their uncertainties were set to $1 \mathrm{~cm}^{-1}$ in order not to interfere with the true experimental values. The QN set comprises eight quantum numbers/labels selected to represent a general rovibronic state of $\mathrm{CaOH}$ as in Tables 4 and 5: vibronic state label, $e / f$ parity, $J, v_{1}, v_{2}, L, v_{3}$, and $F_{1}, F_{2}$.

The MARVEL energy file (output) was processed via the online MARVEL application using the Cholesky (analytic) approach with a $0.05 \mathrm{~cm}^{-1}$ threshold on the uncertainty of the "very bad" lines, which produced a MARVEL energy file containing 1955 states. The MARVEL data set covers rotational excitation up to $J=62.5$ for molecular states below $29,000 \mathrm{~cm}^{-1}$. The MARVEL output and structure of the MARVEL energy levels is illustrated in Table 5 and is also plotted in Figure 2 as a function of $J$.

Figure 3 offers a visual representation of the $\mathrm{CaOH}$ MARVEL network, where the upper state energies are connected with the lower state energies via circles. The size of a circle is $\log (n+1)$, where $n$ is the number of transitions supporting the corresponding upper state. The vertical bars along the horizontal axis show the lower state energies, while the horizontal bars along the vertical axis give the upper state energies. The value of $n$ ranges from 1 (dark blue) to 38 (red).

\section{Conclusions}

We have comprehensively evaluated the published spectroscopic literature on $\mathrm{CaOH}$ and extracted all meaningful

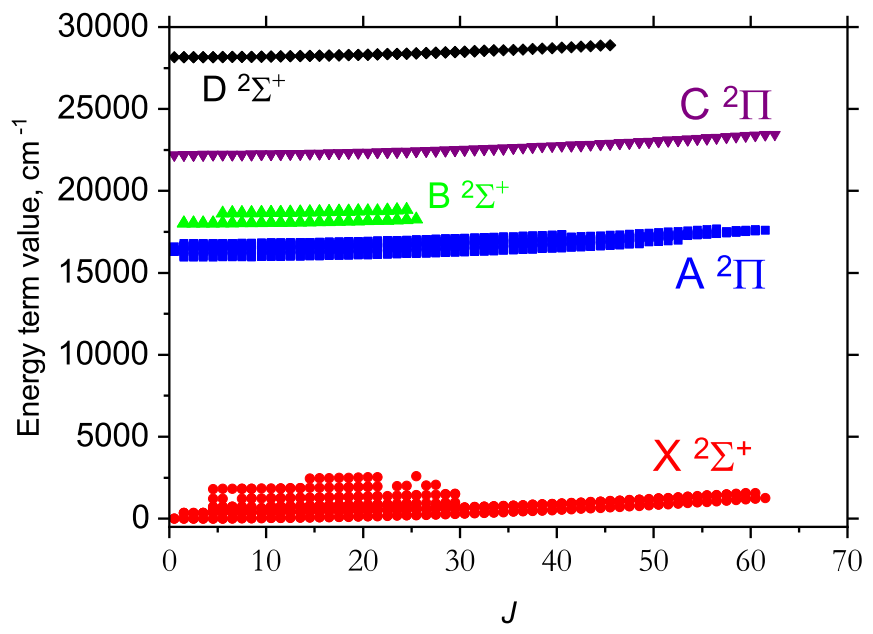

Figure 2. MARVEL energy term values for $\mathrm{CaOH}$ shown for different electronic bands.

molecular rovibronic transition data. These data were analyzed using the robust MARVEL algorithm, which converts assigned transitions into a consistent set of uniquely labeled empirical energy levels with measurement uncertainties. The data set covers rotational excitation up to $J=62.5$ for 1955 molecular states below $29,000 \mathrm{~cm}^{-1}$. The MARVEL input and output files for $\mathrm{CaOH}$ are provided as supplementary material and can be readily updated to include new experimental rovibronic measurements.

While we have analyzed data from five electronic states and a large range of rotational levels, the experimental data has only limited coverage of vibrationally excited states. There is no empirical information on the $\nu_{3}$ stretching vibrational mode and only limited information on vibrational excitation of the other modes in electronically excited states. This means that any line list constructed for this molecule will have to rely on ab initio predictions for the missing quantities.

With the renewed interest in $\mathrm{CaOH}$, we expect the new MARVEL data set to help future detection of this molecule in astronomical environments. The most immediate benefit will be in the calculation of a comprehensive $\mathrm{CaOH}$ molecular line list as part of the ExoMol project (Tennyson \& Yurchenko 2012; Tennyson et al. 2016). A list of highly accurate empirical 


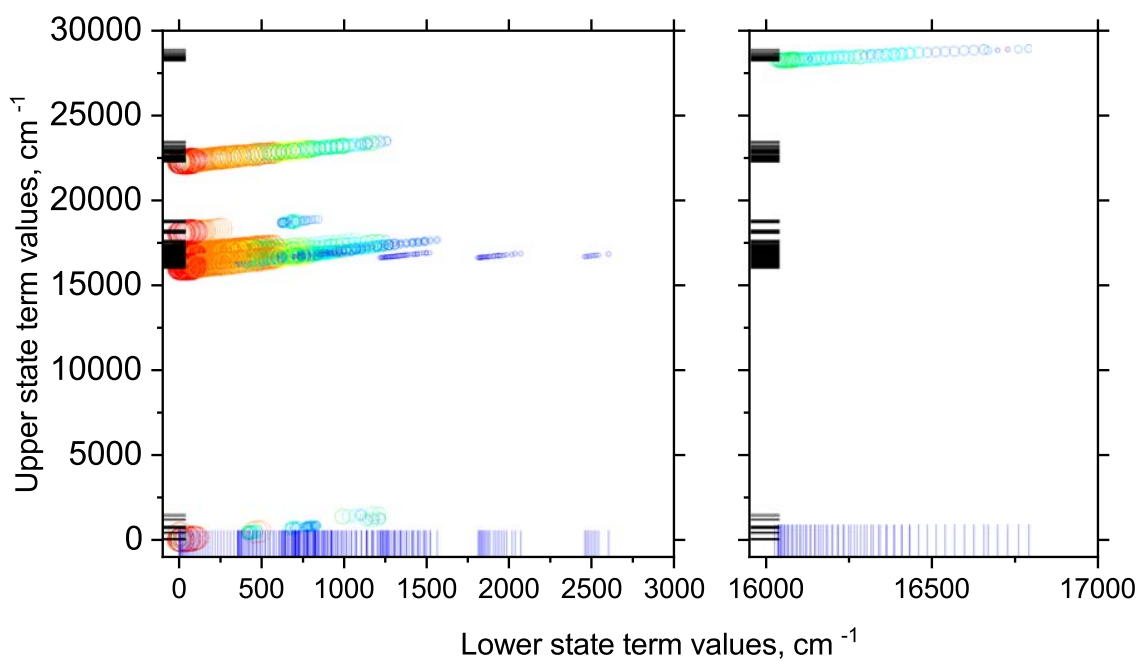

Figure 3. MARVEL network for $\mathrm{CaOH}$ : the upper state energies are plotted against corresponding lower state energies. The vertical bars along the horizontal axis represent the lower state energies, while the horizontal bars along the vertical axis give the upper state energies. Each circle represents a particular transition, with the size proportional to the $\log$ of the number of transitions $n$ plus 1 , going to the upper state. The value ranges from 1 (dark blue) to 38 (red).

energy levels is necessary to refine the theoretical spectroscopic model of a molecule to achieve orders-of-magnitude improvements in the accuracy of the predicted line positions. Accurate molecular opacities of $\mathrm{CaOH}$ are essential for detecting this molecule in hot rocky super-Earth exoplanets and this work brings us closer to this goal.

As mentioned before, a detailed knowledge of the energy level structure in $\mathrm{CaOH}$ will help the design of efficient laser cooling schemes in ultracold molecule research and precision tests of fundamental physics. The alkaline earth monohydroxide radicals are attractive molecules in this pursuit due to their favorable energy level structure, as demonstrated by $\mathrm{SrOH}$, which was the first untrapped polyatomic molecule to be laser-cooled (Kozyryev et al. 2017).

This work was supported by the STFC Project No. ST/ R000476/1. We thank Peter Bernath for collecting experimental data and many helpful discussions. Y.W.'s visit was supported by Physics Boling Class at Nankai University.

Software: MARVEL, PGOPHER.

\section{ORCID iDs}

Yixin Wang (iD https://orcid.org/0000-0001-9286-9501 Alec Owens (i) https://orcid.org/0000-0002-5167-983X Jonathan Tennyson (i) https://orcid.org/0000-0002-4994-5238 Sergei N. Yurchenko (i) https://orcid.org/0000-00019286-9501

\section{References}

Al Derzi, A. R., Furtenbacher, T., Yurchenko, S. N., Tennyson, J., \& Császár, A. G. 2015, JQSRT, 161, 117

Árendás, P., Furtenbacher, T., \& Császár, A. G. 2016, JMaCh, 54, 806 Augustovičová, L. D., \& Bohn, J. L. 2019, NJPh, 21, 103022

Bauschlicher, C. W., Langhoff, S. R., \& Partridge, H. 1986, JChPh, 84, 901 Bauschlicher, C. W., Langhoff, S. R., Steimle, T. C., \& Shirley, J. E. 1990, JChPh, 93, 4179

Bauschlicher, C. W., \& Partridge, H. 1984, CPL, 106, 65

Bernath, P. F. 2009, IRPC, 28, 681

Bernath, P. F., \& Brazier, C. R. 1985, ApJ, 288, 373

Bernath, P. F., \& Kinsey-Nielsen, S. 1984, CPL, 105, 663

Brown, J. M., Hougen, J. T., Huber, K. P., et al. 1975, JMoSp, 55, 500
Bunker, P. R., \& Jensen, P. 1998, Molecular Symmetry and Spectroscopy (2nd ed.; Ottawa: NRC Research Press)

Chubb, K. L., Joseph, M., Franklin, J., et al. 2018a, JQSRT, 204, 42

Chubb, K. L., Naumenko, O. V., Keely, S., et al. 2018b, JQSRT, 218, 178

Coxon, J. A., Li, M. G., \& Presunka, P. I. 1991, JMoSp, 150, 33

Coxon, J. A., Li, M. G., \& Presunka, P. I. 1992, MolPh, 76, 1463

Coxon, J. A., Li, M. G., \& Presunka, P. I. 1994, JMoSp, 164, 118

Császár, A. G., Czakó, G., Furtenbacher, T., \& Mátyus, E. 2007, Annu. Rep. Comput. Chem., 3, 155

Császár, A. G., \& Furtenbacher, T. 2011, JMoSp, 266, 99

Darby-Lewis, D., Shah, H., Joshi, D., et al. 2019, JMoSp, 362, 69

Dick, M. J., Sheridan, P. M., Wang, J. G., Yu, S., \& Bernath, P. F. 2006, JMoSp, 240, 238

Duley, W. W., \& Millar, T. J. 1978, ApJ, 220, 124

Endres, C. P., Schlemmer, S., Schilke, P., Stutzki, J., \& Müller, H. S. P. 2016, JMoSp, 327, 95

Fegley, B., Jr., Jacobson, N. S., Williams, K. B., et al. 2016, ApJ, 824, 103

Furtenbacher, T., Árendás, P., Mellau, G., \& Császár, A. G. 2014, NatSR, 4, 4654

Furtenbacher, T., \& Császár, A. G. 2012, JMoSt, 1009, 123

Furtenbacher, T., Császár, A. G., \& Tennyson, J. 2007, JMoSp, 245, 115

Furtenbacher, T., Szabó, I., Császár, A. G., et al. 2016, ApJS, 224, 44

Furtenbacher, T., Szidarovszky, T., Fábri, C., \& Császár, A. G. 2013a, PCCP, 15,10181

Furtenbacher, T., Szidarovszky, T. M., Edit Fábri, C., \& Császár, A. G. 2013b, J. Chem. Theory Comput., 9, 5471

Hailey, R. A., Jarman, C., \& Bernath, P. F. 1997, JChPh, 107, 669

Hilborn, R. C., Qingshi, Z., \& Harris, D. O. 1983, JMoSp, 97, 73

Hoeijmakers, H. J., de Kok, R. J., Snellen, I. A. G., et al. 2015, A\&A, 575, A20

Hougen, J. T. 1962a, JChPh, 36, 1874

Hougen, J. T. 1962b, JChPh, 37, 403

Hougen, J. T. 1962c, JChPh, 36, 519

Jarman, C. N., \& Bernath, P. F. 1992, JChPh, 97, 1711

Jungen, C. 2019, JMoSp, 363, 111172

Kong, J., \& Boyd, R. J. 1996, JChPh, 104, 4055

Koput, J., \& Peterson, K. A. 2002, JPCA, 106, 9595

Kozyryev, I., Baum, L., Matsuda, K., et al. 2017, PhRvL, 118, 173201

Kozyryev, I., Steimle, T. C., Yu, P., Nguyen, D.-T., \& Doyle, J. M. 2019, NJPh, 21, 052002

Li, M. G., \& Coxon, J. A. 1992, JChPh, 97, 8961

Li, M. G., \& Coxon, J. A. 1995, JChPh, 102, 2663

McKemmish, L. K., Borsovszky, J., Goodhew, K. L., et al. 2018, ApJ, 867, 33

McKemmish, L. K., Masseron, T., Hoeijmakers, J., et al. 2019, MNRAS, 488, 2836

McKemmish, L. K., Masseron, T., Sheppard, S., et al. 2017, ApJS, 228, 15

Müller, H. S. P., Schlöder, F., Stutzki, J., \& Winnewisser, G. 2005, JMoSt, 742,215

Müller, H. S. P., Thorwirth, S., Roth, D. A., \& Winnewisser, G. 2001, A\&A, 370, L49

Mulliken, R. 1931, RvMP, 3, 0089 
Ortiz, J. V. 1990, JChPh, 92, 6728

Pesch, P. 1972, ApJL, 174, L155

Pople, J. 1960, MolPh, 3, 16

Rajpurohit, A. S., Reyle, C., Allard, F., et al. 2013, A\&A, 556, A15

Rivlin, T., Lodi, L., Yurchenko, S. N., Tennyson, J., \& Le Roy, R. J. 2015, MNRAS, 451, 634

Schaefer, L., Lodders, K., \& Fegley, B., Jr. 2012, ApJ, 755, 41

Scurlock, C. T., Fletcher, D. A., \& Steimle, T. C. 1993, JMoSp, 159, 350

Taylor, C. M., Chaudhuri, R. K., \& Freed, K. F. 2005, JChPh, 122, 044317

Tennyson, J., Bernath, P. F., Brown, L. R., et al. 2009, JQSRT, 110, 573

Tennyson, J., Bernath, P. F., Brown, L. R., et al. 2010, JQSRT, 111, 2160

Tennyson, J., Bernath, P. F., Brown, L. R., et al. 2013, JQSRT, 117, 29

Tennyson, J., Bernath, P. F., Brown, L. R., et al. 2014a, Pure Appl. Chem., 86,71

Tennyson, J., Bernath, P. F., Brown, L. R., et al. 2014b, JQSRT, 142, 93

Tennyson, J., \& Yurchenko, S. N. 2012, MNRAS, 425, 21
Tennyson, J., \& Yurchenko, S. N. 2017, MolAs, 8, 1

Tennyson, J., Yurchenko, S. N., Al-Refaie, A. F., et al. 2016, JMoSp, 327, 73

Theodorakopoulos, G., Petsalakis, I. D., Liebermann, H. P., Buenker, R. J., \& Koput, J. 2002, JChPh, 117, 4810

Tóbiás, R., Furtenbacher, T., Császár, A. G., et al. 2018, JQSRT, 208, 152

Tóbiás, R., Furtenbacher, T., Tennyson, J., \& Császár, A. G. 2019, PCCP, 21,3473

Tsuji, T. 1973, A\&A, 23, 411

Watson, J. K. 1970, MolPh, 19, 465

Western, C. M. 2017, JQSRT, 186, 221

Yurchenko, S. N., Williams, H., Leyland, P. C., Lodi, L., \& Tennyson, J. 2018, MNRAS, 479, 1401

Ziurys, L. M., Barclay, W. L., \& Anderson, M. A. 1992, ApJL, 384, L63

Ziurys, L. M., Fletcher, D. A., Anderson, M. A., \& Barclay, W. L. 1996, ApJS, 102,425 